\title{
5 \\ Air Operations Control and Reporting Centre
}

Chris Westwood

Much had happened in Afghanistan following the withdrawal of the Special Forces Task Group in 2002. Australian land forces returned in 2005. Thereafter calls were being made for additional niche contributions, including critical capabilities in relatively short supply. This included calls for capabilities to manage the increasingly busy airspace over Afghanistan, such as the RAAF's Control and Reporting Centre (CRC), a field deployable unit designed to manage the use of airspace in a war zone.

The 2007-09 deployment of the RAAF's CRC was drawn from No. 41 Wing, Surveillance and Response Group (SRG). The CRC was responsible for deconflicting civilian and military air traffic over Afghanistan as well as ensuring that all military aircraft, both manned and remotely piloted, were properly marshalled within the area of operations. The CRC deployment represented only one small but important part of a significant RAAF contribution to operations during this period. This contribution included the following elements:

- Air Mobility-86 Wing (C-130 Hercules air transport and air-to-air refuelling aircraft)

- Heron unattended aerial vehicles-5 Flight (used for reconnaissance and surveillance)

- APC3 Orion surveillance aircraft-92 Wing (used for maritime as well as land surveillance over Iraq from 2005 onwards) 
- Air Traffic Control—44 Wing (personnel deployed at various airfields alongside US and coalition counterparts)

- An Air Task Group, consisting of the following two components:

1. Air Combat-81 Wing (F/A-18 Hornet fighter aircraft deployed particularly for Operation FALCONER in 2003 over Iraq)

2. Air-to-Air Refuelling-33 Squadron (operating Boeing 707 and later KC-30 refuelling aircraft throughout the Persian Gulf region)

- Intelligence-87 Squadron (collection, analysis and reporting for deployed force elements)

- Medical-Aeromedical Evacuation, Role 2 (deployed with the land forces in Tarin Kowt, Uruzgan)

- Joint Terminal Attack Controller-4 Squadron (providing precision targeting assistance to deployed forces)

- Combined Air Operations Centre (CAOC; RAAF contribution comprising personnel assigned to various command, operations, intelligence and logistics appointments alongside US Air Force and other coalition personnel managing coalition air operations across the Middle East)

- Various units delivering communications, combat support and supplementing joint staff appointments

- Control and Reporting Centre (CRC)—41 Wing.

In short, for the period 2001-14, lots of great Air Force people were doing great things. The forthcoming official histories will provide coverage of the breadth and depth of the RAAF's operations in the Middle East. ${ }^{1}$ So I will simply acknowledge the great achievements of the RAAF in general, and individual members in particular, and move on to the CRC.

A niche deployment such as the CRC is generally overshadowed in discussions regarding air power by fast jets and bombs. Perhaps not surprisingly given my background, I believe there is much to be learned from examination of these smaller capabilities such as the CRC. And lessons drawn from such examination are relevant not just to CRC or even broader command, control, communications, computer, intelligence, surveillance and reconnaissance (C4ISR) operations, but also for all small, high-end niche capabilities.

1 See Australian War Memorial, 'Historians: Official History of Australian operations in Iraq, Afghanistan and East Timor' at www.awm.gov.au/learn/understanding-military-history/officialhistories/iraq-afghanistan-timor/oh (retrieved 21 October 2020). 


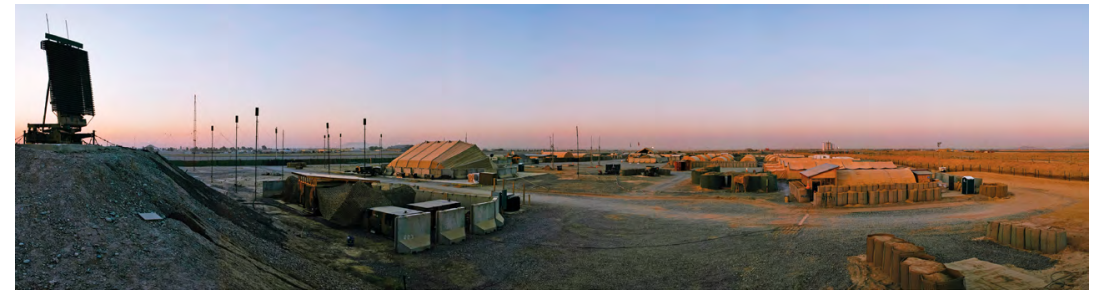

RAAF Control and Reporting Centre, Kandahar, 2007-09.

Source: Courtesy of Air Commodore Chris Westwood.

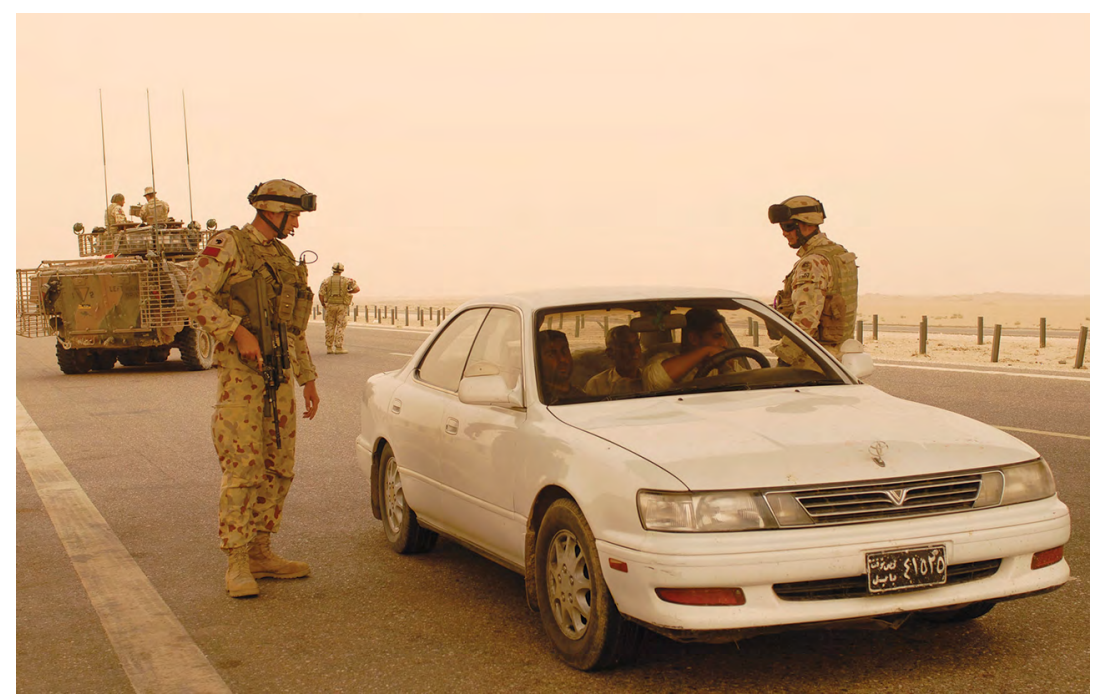

Australian soldeirs check vehicles on a main supply route in Southern Iraq, 2008.

Source: Courtesy of the Department of Defence.

The CRC by definition includes sensors - in this case our own Lockheed Martin TPS-77 radar, plus access to all other deployed radars; an operations facility-contained within a series of ISO containers inside a large tent; and some link (military tactical data exchange networked communications) capabilities and facilities both at the CRC site and scattered around the theatre. The CRC compiled an air picture characterising the Afghanistan airspace from this facility and provided that to various agencies, primarily the CAOC. The CRC also used their air picture to provide 24/7 air battle management throughout the Afghanistan airspace. 
The role of the CRC is simple: to ensure we have the right aircraft with the right weapons and fuel load in the right place at the right time to support the joint (interservice) fight-our priority was to support the troops in contact with adversaries.

This was the first RAAF CRC offshore operational deployment in 50 years. It was fair to say that at the time, while we were good at moving capabilities around Australia, we had little offshore deployment experience or culture, and operating inside a deployed Joint Task Force (JTF) was reasonably foreign. So we had a steep learning curve. However, there was no better operational or technical CRC capability of its type at the time on the planet-including the operators and technicians (and support staff) who were, and still are, highly trained.

In terms of operational statistics, there were 196,000 sorties that came through the CRC in the 23-month deployment, more than any operator would see in a full career. The CRC supported 7,000 troops in contact during that time-something we are particularly proud of. This was a busy operational deployment in the busiest airspace in the world at the time.

In addition to these key operational achievements, there were some impressive technical statistics as well. In the 23 months of operations, the capability operated $24 / 7$, achieving close to 100 per cent availability. This was a big ask for this type of sensitive electronic equipment, which included many single points of failure, operating in such a harsh climatic zone and under considerable operational pressure. Credit needs to go to our outstanding technical teams who took these challenges in their stride.

Figure 1 shows the airspace divisions in Afghanistan at the time of the RAAF CRC deployment. There were three Air Battle Management agencies controlling three Afghanistan Battle Management Areas (BMAs): an RAF CRC ('Crowbar'), a US AWACS ('Wizard') and our RAAF CRC ('Taipan'). The RAAF CRC was the primary agency and had Operational Control (OPCON) of the RAF CRC and US AWACS. Effectively, the RAAF CRC 'controlled' the Afghanistan skies for 23 months. In the context of the ADF, this represents a historically significant achievement. 


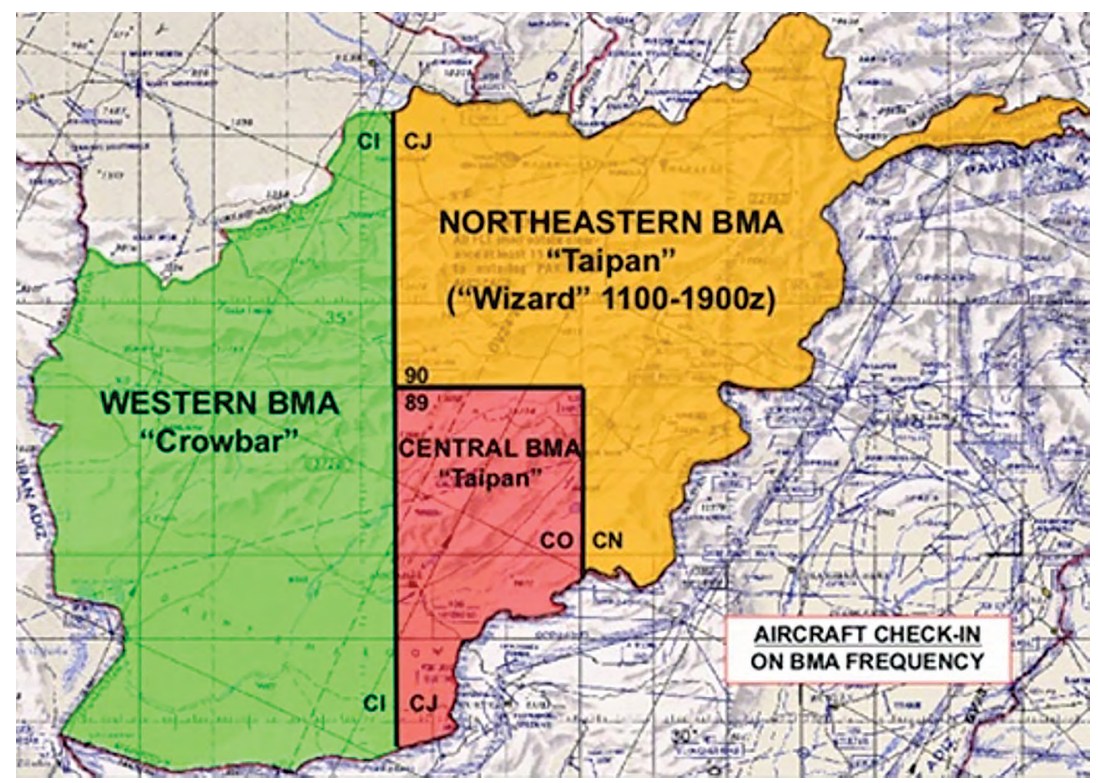

Figure 1: Afghanistan airspace.

Source: Courtesy of Air Commodore Chris Westwood.

\section{Lessons}

There were myriad lessons at all levels and from many perspectives concerning this deployment. In compiling these lessons, I spent a bit of time with many of the key players involved in the deployment. In particular, I would like to acknowledge Group Captain Richard Pizzuto. Richard was one of the deployment commanders and maintains a keen interest in capturing the history of the CRC deployment. I have collated these lessons into strategic, operational and tactical categories and listed the top ones of each.

Figure 2 shows a simplified command and control (C2) diagram. Operationally, the CRC worked for the CAOC. Everything was real time, which challenged those well-intentioned staff officers who were used to making decisions in days with well-crafted decision briefs. 


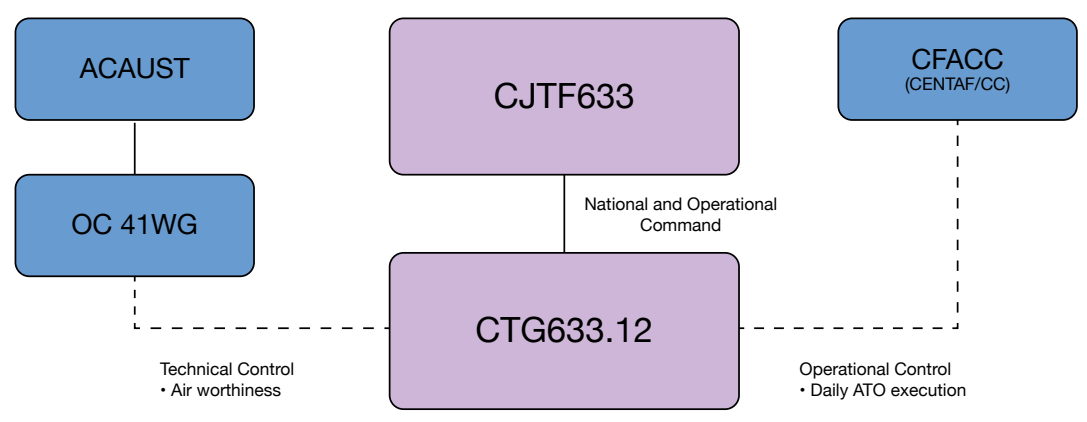

Figure 2: Control and Reporting Centre command and control arrangements.

Source: Courtesy of Air Commodore Chris Westwood.

\section{Strategic lessons}

The value of high-end niche capabilities such as this to Australia's alliance relationship with the United States cannot be overstated. US CRC capabilities were very tired, technically and personally. US Marines and USAF personnel had many rotations since 2001. Even in major forces, the pool of personnel and equipment available to service deployments of this type is limited and can be overused. Frankly, the US people and equipment were looking for an opportunity to reconstitute the force. Deploying the RAAF CRC into Kandahar for two years was a great way to give our US counterparts a well-earned and much-needed break-and they were extremely grateful.

Being offered a significant $\mathrm{C} 2$ role in a combined force, such as the Air Battle Management OPCON role of the CRC, with so much direct operational interaction with allied forces, demonstrated the trust the United States had in our CRC. Our operators and commanders had to be known and trusted by our allies, with compatible if not common doctrine and procedures. Things such as Air Battle Management, tanking (refuelling) procedures, 'Link 16' data communications doctrine and protocols and so on cannot be learnt on the fly. This underscores the importance of investment in high-end exercises to continually prepare and develop forces, and expose personnel to partners. Exercises such as Red Flag, where high-end capabilities can be tested and integrated with allies and put on show, are critical. Australian exercises need to be complex and attractive to the full range of partners, and need to include genuine, demanding, warlike scenarios. Trust is critical. 
There is also a need to invest in education. Broad education among the key stakeholders can make or break a deployment. Very few people in the ADF, at all levels, understood the nuances of the CRC capabilitywhich is perfectly understandable. The same, no doubt, is true for many current high-end niche capabilities such as electronic warfare, cyber and information operations for instance. More time spent demonstrating the CRC capability, and explaining where the 'one sizes fits all' mentality might not work, would have been useful. Niche technical capabilities of this type tend to be locked up and somewhat hidden behind secure doors. This is not always helpful. Everyone we depend on to make operational deployments successful needs to understand what these niche capabilities do and what their unique requirements might be.

Most important of all is relationships, of course. Knowing and trusting each other at all levels fixes most problems. Having RAAF officers as Deputy Combined Forces Air Component Command (CFACC) and CAOC directors, as well as valuing long-standing relationships between senior officers throughout the RAAF, the Australian Army and Navy, US forces and RAF, was a great aid in working through local issuesincluding getting the local C2 squared away. Likewise, relationships in Headquarters Joint Operations Command (HQJOC) at all levels helped work through many teething issues. Relationships always have been and always will be the key to success. Knowing each other through courses, exchanges and exercises can create the rapport and trust necessary to overcome problems at all levels.

\section{Operational lessons}

As I have mentioned, Afghanistan was the first deployment of the RAAF for 50 years. We, of course, had moved the CRC capability around Australia on many occasions, which did develop some reasonable technical deployment skills, but it was clear that some of the more JTF-level skills needed to be developed. The CRC team learnt quickly, but there were tears along the way.

For example, the CRC did not engage the JTF staff effectively in the pre-deployment phase. Hence JTF personnel did not understand the CRC's deployment needs, and CRC personnel did not understand their processes. The result was that pre-deployment reconnaissance was somewhat underdone. What should have been a dedicated activity was instead tagged on to a special forces reconnaissance, which clearly had 
different priorities. The CRC Recon Team was subsequently slashedeven losing the logistics officer. Deployment planning therefore became that much harder, and the advance party ultimately had a more difficult task as a result.

The CRC also had an unusual in-theatre training and assessment requirement for operators, which the JTF personnel staff never did quite get to grips with. This was the busiest operational airspace in the world. There was no way to simulate the intensity. None of the team had experienced the sheer volume of aircraft and the absolute urgency of operations. The Mission Rehearsal Exercise (MRE) that was developed by 41 Wing was as good as it could be, based on in-theatre Air Task Orders and Airspace Control Orders, and using a representative simulation capability. It was good enough for the USAF to adopt, but it still could not replicate the intensity or tempo of real operations. As such, operators were put through a $2-3$-week training program on arrival in theatre before facing an assessment that, if passed, certified them for live operations. Unfortunately, a handful of operators (about eight over the 23 months of the deployment) could not make the jump from MRE to live operations, and they were tagged for compulsory return to Australia. This invariably invoked lengthy engagements with the JTF personnel and legal staff, who did not appreciate the due process and rigour that had already been applied; sometimes they insisted on adding their own review. Such reviews might take a week, during which time the CRC would be short an operator.

Being something of an unknown capability to many in the JTF and HQJOC, the CRC always forced an uphill battle to justify its existence or, more precisely, its workforce. This was a particular issue in Afghanistan, where the government imposed a hard limit on the number of 'boots on the ground'. The CRC had a 75-person footprint that replaced a US footprint approximately twice the size. That is not unusual. The ADF has long prided itself on multiskilling and doing more with less. This reflects the demands of operating a mid-size force with best of breed equipment. It seems fine on paper until illness strikes or when someone does not come up to speed and needs to be replaced. There were unique challenges in this regard, yet the operational personnel allocation table was considered sacrosanct. The concept of 'one size fits all' is outdated and largely irrelevant in a modern force. 
Finally, at the operational level, I must mention the redeployment. 41 Wing (under the TECHCON umbrella) had planned the extrication of the CRC in detail to ensure the protection of millions of dollars' worth of equipment from packing and handling mistakes. The wing's concerns were not shared by the JTF staff, however, who insisted yet again on applying the standard model, a model that did not include the deployment of a specialist pack-up team as planned by the wing. The resulting damage to the $\$ 20$ million radar when it struck the boom gate as it was driven off the site by a contractor easily accounted for any savings garnered by the decision not to deploy the specialist 41 Wing team.

\section{Tactical lessons}

Onelesson at the tactical level concerned workforce sustainment. The CRC initially deployed for two rotations and one 12-month deployment. Once the deployment of the wing was extended (no real surprise), there were numerous workforce sustainment and concurrency issues, many unique to small, highly specialised capabilities of this type. My message is twofold: first, there is a clear need to be flexible and innovative as a whole force with workforce sustainment (medical, rotation durations and so on), and second, there is a need to listen to those who understand the specific workforce pressures of these capabilities. One size does not always fit all.

\section{Summary}

Overwhelmingly, this was a great deployment that achieved great results, and one of which those involved can be proud. That is important. The commitment and professionalism shown by the 400 or so ADF members who deployed with the CRC was yet another example of the remarkable people who serve in the ADF. And finally, there is plenty to learn about deploying niche capabilities. These will become more and more crucial to the joint and integrated force as fifth-generation technology takes hold. The lessons drawn from the CRC deployment are relevant for future highend niche capabilities, be they cyber, electronic warfare or whatever comes next. So we should not be shy about discussing them now. 
This text is taken from Niche Wars: Australia in Afghanistan and Iraq, 2001-2014, edited by John Blaxland, Marcus Fielding and Thea Gellerfy, published 2020 by ANU Press, The Australian National University,

Canberra, Australia.

doi.org/10.22459/NW.2020.05 